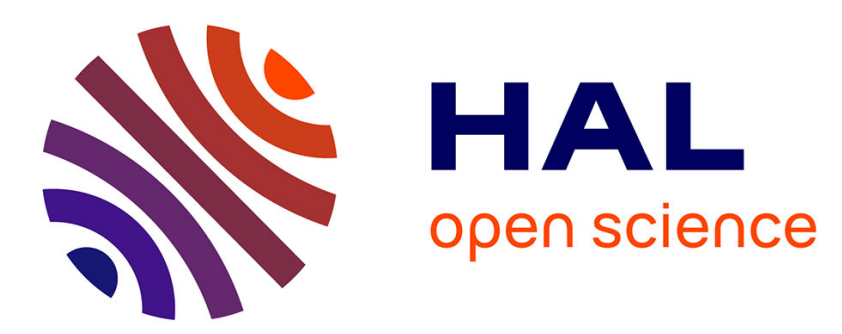

\title{
Tests and foreseen developments of fibered-OSLD gamma heating measurements in low-power reactors
}

\author{
A. Gruel, M. Le Guillou, Patrick Blaise, C. Destouches, Sylvain Magne
}

\section{To cite this version:}

A. Gruel, M. Le Guillou, Patrick Blaise, C. Destouches, Sylvain Magne. Tests and foreseen developments of fibered-OSLD gamma heating measurements in low-power reactors. ANIMMA $2017-$ Advancements in Nuclear Instrumentation Measurement Methods and their Applications, Jun 2017, Liège, Belgium. pp.04010, 10.1051/epjconf/201817004010 . cea-01772739

\section{HAL Id: cea-01772739 https://hal-cea.archives-ouvertes.fr/cea-01772739}

Submitted on 18 Jan 2020

HAL is a multi-disciplinary open access archive for the deposit and dissemination of scientific research documents, whether they are published or not. The documents may come from teaching and research institutions in France or abroad, or from public or private research centers.
L'archive ouverte pluridisciplinaire HAL, est destinée au dépôt et à la diffusion de documents scientifiques de niveau recherche, publiés ou non, émanant des établissements d'enseignement et de recherche français ou étrangers, des laboratoires publics ou privés. 


\title{
Tests and foreseen developments of fibered- OSLD gamma heating measurements in low- power reactors
}

\author{
A. Gruel, M. Le Guillou, P. Blaise, C. Destouches, S. Magne
}

\begin{abstract}
In this paper are presented test measurements of a fibered-OSLD system performed during a dedicated experimental phase in EOLE zero-power reactor. The measurement setup consists of an OSLD crystal connected onto the extremity of an optical fiber and a laser stimulation system, manufactured by the CEA/LIST in Saclay. The OSL sensor is remotely stimulated via an optical fiber using a diode-pumped solid-state laser. The OSL light is collected and guided back along the same fiber to a photomultiplier tube. Results obtained using this system are compared to usual gamma heating measurement protocol using OSLD pellets. The presence of induced radio-luminescence in the OSLD during the irradiation was also observed and could be used to monitor the gamma flux.

The feasibility of remote measurements is achieved, whereas further developments could be conducted to improve this technique since the readout procedure still requires to withdraw the OSLD off the gamma flux (hence from the core) on account of the dose rate (around a few $G y \cdot h^{-1}$ ), and the readout time remains quite long for on-line applications. Several improvements are foreseen, and will be tested in the forthcoming years.
\end{abstract}

Index Terms - nuclear heating, optical fibers, optically stimulated luminescent dosimeters, remote readout

\section{INTRODUCTION}

$\mathrm{T}$ HE accurate determination of nuclear heating in materials is a major issue for the development of nuclear technology and in particular the design of new core concepts, such as the Jules Horowitz material testing reactor [1].

In material testing reactors the neutron and gamma flux levels allow to measure directly the nuclear heating by calorimetry based techniques for instance. In mock-ups or zero-power reactors (ZPRs), such as EOLE and MINERVE reactors at Cadarache [2], the low operating level - typically from some watts to some kilowatts - requires the use of different methods such as dosimetry. In the past few years, several experimental programs [3][4] were devoted to the improvement of gamma heating measurements using thermoluminescent and optically stimulated luminescent dosimeters

Submitted on $01 / 06 / 2017$

A. Gruel, M. Le Guillou, P. Blaise and C. Destouches are with CEA, DEN, DER, Experimental Physics Service, F-13108 Saint Paul lez Durance, France (e-mail: adrien.gruel@cea.fr).

S. Magne is with CEA, LIST, Laboratoire Capteurs Architectures Électroniques, F-91191 Gif-sur-Yvette, France.
(TLDs and OSLDs, respectively). However, the main drawback of nuclear heating measurement in low-power reactors remains the off-line gamma heating estimation, given that both TLD and OSLD stimulations (thermal and optical respectively) allowing the readout of the absorbed doses can only be performed after irradiation and out of core. To circumvent this disadvantage, an effort is being made on the development of in situ and on-line measurement of the gamma heating. One of the considered solutions is the use of optical fibered systems.

In the first section are presented the main principles of gamma heating measurements in low-power reactors, and especially the system that was used for. In the second section are presented the results of test measurements performed in the EOLE reactor.

\section{GAMMA HEATING MEASUREMENTS USING OPTICAL FIBERS}

\section{A. Overview and general principles}

Nuclear heating in material arises from the local deposition of energy carried by neutrons, prompt photons issued from fissions, radiative captures and inelastic neutron scattering, and delayed photons emitted by fissions and activation product decays. This energy is transferred to the electrons through neutral particle interactions, and finally deposited in the material.

Among the different existing techniques, two are particularly suitable for gamma heating measurements in ZPR: thermo-luminescent dosimetry (TLD) and optically stimulated luminescent dosimetry (OSLD). Both techniques are based on the luminescence emitted by the recombination of trapped electrons after a thermal or optical stimulation of the detector. Usually TLDs and OSLDs are small pellets of luminescent material with high photon sensitivity stacked in metal pillboxes. The main drawback is that the readout process is performed offline, out of core.

In order to allow a remote and/or on-line measurement of the gamma heating in the core, different types of fibered setups are also based on the same physical principles, the optical fiber carrying both the light or thermal stimulation for the readout procedure and the TL or OSL signal emitted consequently. A comprehensive review of gamma dosimetry techniques can be found in [5]. 


\section{B. Description of the fibered-OSLD system}

A complete description of the system can be found in [6][7], its main principles are summed up hereafter. An overview of the system can be seen Fig. 1. The OSL reader includes a 16channel optical fiber switch actuated by a stepper motor, an optical case and power/USB electronics. The OSL reader is linked to a laptop handled through dedicated software. An OSL fiber sensor consists of an optical fiber cable (armoured with iron thread) with an SMA connector at one end and a steel case at the other. The steel case is rigidly fixed onto the cable extremity. It protects the OSL crystal $\left(\mathrm{Al}_{2} \mathrm{O}_{3}: \mathrm{C}\right.$ crystals, $\varnothing 1 \mathrm{~mm}, 1 \mathrm{~mm}$ long) affixed near the end of a silica fiber and provides a stable coupling of light. The sensors exhibit circular symmetry along the fiber/crystal axis.

After irradiation, each OSL sensor is remotely stimulated using a continuous wave (CW) diode-pumped solid-state laser (@,532 nm, $200 \mathrm{~mW}$ initially) and bleached for a next use. The reader works in the $\mathrm{CW}$ mode (i.e. CW-OSL). The OSL resulting from the stimulation of the crystal, emitted at $410 \mathrm{~nm}$, is then collected by the optical fiber, separated by a dichroic beam-splitter, filtered and eventually detected by a photomultiplier in photon-counting mode.

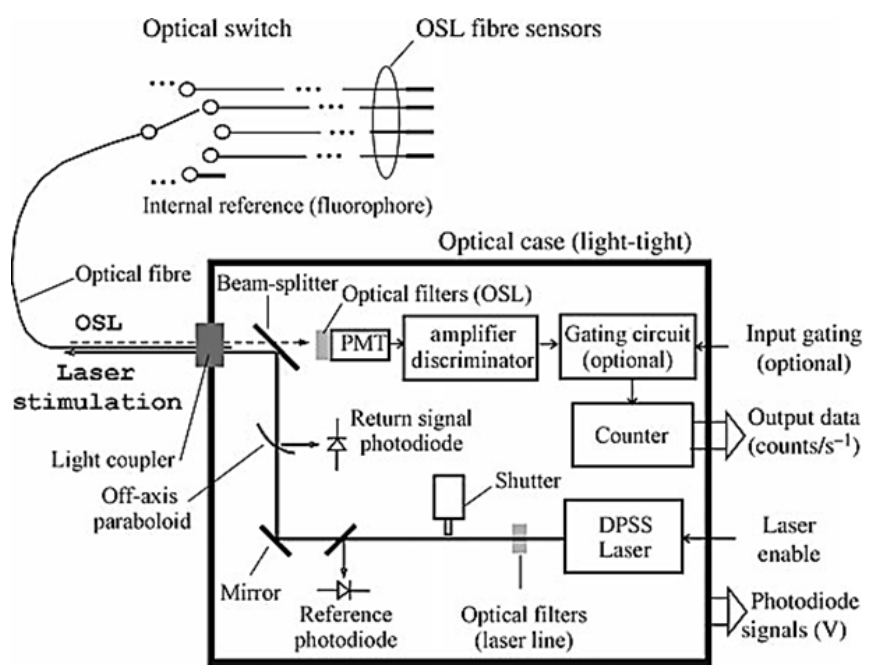

Fig. 1. Schematic of the fibered-OSL reader of the CEA/LIST [6].

\section{TEST MEASUREMENTS IN THE EOLE REACTOR}

\section{A. Absolute dose calibration}

The first step in gamma dose measurements is to characterize the detector response to the absorbed gamma dose. Detectors were calibrated in a pure gamma field at a distance of $80 \mathrm{~cm}$ of a ${ }^{60} \mathrm{Co}$ source, whose $\beta^{-}$decay leads to the emission of two gamma rays at 1.17 and $1.33 \mathrm{MeV}$. The three sensors show the same behavior with significantly different sensitivities, and a second order polynomial was adjusted to the data (Fig. 2).

For comparison measurements in EOLE, OSLD pellets (alumina encapsulated in polyester, from Landauer [8]) stacked in stainless-steel pillboxes were also calibrated in a pure gamma field, up to an integrated dose of 2 Gy. Their responses are linear.

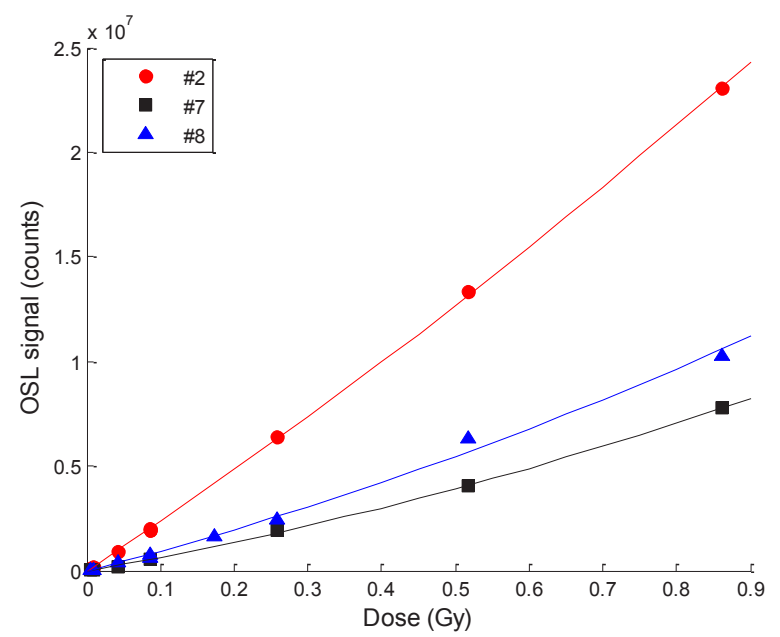

Fig. 2. OSL signal versus dose for the three fibered-OSL sensors (markers) and calibration curves (lines).

\section{B. Experimental setup}

This system was tested in the EOLE reactor. In order to reach better statistics, measurements were carried out with the most sensitive fibered OSL sensor (\#2). The reader was located on the top of the EOLE core, the fiber going inside the core through a plastic tube connected at the upper end of an aluminum overclad.

The OSLD crystal connected to the fiber was set slightly above the core mid-plane, maintained in position by an aluminum shim. Below this sensor, two Landauer nanoDots OLSD pellets in a stainless-steel pillbox were used for comparison purpose, as long as other ones in an equivalent position, but without the fiber atop of them (Fig.3).

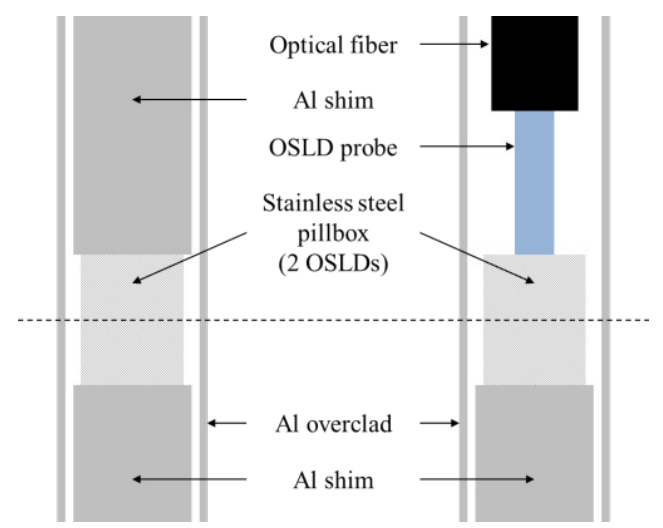

Fig. 3. Schematic axial cut of the detectors localization in the core (dashedline: mid-core plane). Inside the overclad is air, outside is air (background measurement) or borated water (irradiation).

\section{Results and discussion}

The results for the comparison between the fibered-OSLD and the OSLD pellets are shown in Table I. Measurements carried out during irradiations show a very good agreement, 
similar to their respective uncertainties. The discrepancy between the OSLD pellets and the fibered-OSLD in the same channel is $3.5 \%$, while the difference between the fiberedOSLD and the OSLD pellets in the equivalent position is $0.1 \%$. However, the fibered-OSLD was only calibrated up to $0.9 \mathrm{~Gy}$, so the absolute dose value at 1.2 Gy could slightly be biased. Complementary calibration measurements to higher doses must be carried out to obtain a more accurate value.

TABLE I

COMPARISON BETWEEN OSLD PELLETS AND THE FIBERED-OSLD SYSTEM (UNCERTAINTIES AT 1 STANDARD DEVIATION).

\begin{tabular}{cc}
\hline \hline Detector & Irradiation \\
\hline $\begin{array}{c}\text { fibered-OSLD } \\
\text { OSLD pellets in same channel as } \\
\text { fibered-OSLD } \\
\text { OSLD pellets in equivalent } \\
\text { position }\end{array}$ & $1197 \pm 33 \mathrm{mGy}$ \\
\hline \hline
\end{tabular}

Another interesting feature of the fibered-OSL system is the presence of a radio-luminescence (RL) signal during the irradiations. However, in order to use this signal for dose or dose rate measurements, it would require a calibration of the $\mathrm{RL}$ response of the sensors with respect to dose and to dose rate [9].

Nevertheless, some limitations appeared when using this system in EOLE. The main one was that the read-out time of the fibered sensor, from the start of the laser stimulation to the bleaching of the OSL crystal, is similar to the irradiation duration. Another improvement could be to enable the readout of the OSL during irradiation, which requires the increase of the laser power.

\section{CONCLUSION AND OUTLOOKS}

Remote gamma heating measurements were performed in the EOLE reactor using a fibered setup. Standard OSLD pellets were also used for comparison purpose during the irradiations in the core.

The two measurement techniques are in good agreement (within a few percent), and the reproducibility of the measurements of the fibered-OSLD was measured around $3 \%$.

Further development could improve the use of this remote system in ZPRs, such as the decrease of the readout time down to a few seconds, the characterization of the behavior of the detector when the readout is performed during irradiation, and the calibration and the use of the RL signal observed.

\section{ACKNOWLEDGMENTS}

The authors wish to acknowledge the operator team of the EOLE reactor, P.-G. Allinei and P. Chaussonnet (CEA/DEN/DTN/SMTA/LMN) for their participations to the calibration campaign, as well as L. De Padua and N. Makaryan (CEA/DEN/D2S/SPR/LRIR).

\section{REFERENCES}

[1] M. Lemaire et al., Experimental validation of photon-heating calculation for the Jules Horowitz Reactor, Nucl. Instr. Meth. A (2015), Vol. 780, pp. 68-80DOI: 10.1016/j.nima.2015.01.054

[2] J.-C. Bosq et al., The Use of EOLE and MINERVE Critical Facilities for the Generation 3 Light Water Reactors Studies, RGN (2011), Vol. 5, pp. 63-69. DOI: $10.1051 / \mathrm{rgn} / 20115063$

[3] D. Blanchet, Ph.D. thesis, Université Blaise Pascal, France, 2006

[4] H. Amharrak, Ph.D. thesis, Aix-Marseille Université, France, 2012

[5] M. Le Guillou et al., State of the art on nuclear heating measurement methods and expected improvements in zero power research reactors, EPJ Nuclear Sci. Technol. (2017), Vol. 3, No. 11, DOI: 10.1051/epjn/2017002

[6] S. Magne et al., Multichannel dosemeter and Al2O3:C optically stimulated luminescence fibre sensors for use in radiation therapy: evaluation with electron beams, Radiat. Prot. Dosim. (2008), Vol. 131, No. 1, pp. 93-99. DOI: 10.1093/rpd/ncn226

[7] S. Magne et al., Multichannel Dosimeter and $\alpha-\mathrm{Al}_{2} \mathrm{O}_{3}: \mathrm{C}$ Optically Stimulated Luminescence (OSL) Fiber Sensors for Use in Radiation Therapy-Evaluation With Photon Beams, IEEE Trans. Nucl. Sci. (2011), Vol. 58, No. 2, pp. 386-394, DOI: 10.1109/TNS.2011.2114369

[8] C. A. Perks et al., Medical dosimetry using Optically Stimulated Luminescence dots and microStar readers, in $12^{\text {th }}$ Int. Congr. Int. Radiation Protection Assoc., Buenos Aires, Argentina, 2008

[9] S. Magne et al., Fiber-coupled, time-gated $\mathrm{Al}_{2} \mathrm{O}_{3}: \mathrm{C}$ radioluminescence dosimetry technique and algorithm for radiation therapy with LINACs, IEEE Trans. Nucl. Sci. (2013), Vol. 60, No. 4, pp. 2998-3007, DOI: 10.1109/TNS.2013.2263640 